\title{
Intramyocardial thrombin promotes angiogenesis and improves cardiac function in an experimental rabbit model of acute myocardial infarction
}

\author{
Sofoklis Mitsos, PhD, ${ }^{a}$ Efstratios N. Koletsis, $\mathrm{PhD},{ }^{\mathrm{a}}$ Konstantinos Katsanos, $\mathrm{PhD},{ }^{\mathrm{b}}$ Vassiliki Bravou, $\mathrm{PhD},{ }^{\mathrm{c}}$ \\ Fevronia Kolonitsiou, $\mathrm{PhD},{ }^{\mathrm{d}}$ Evangelos Marinos, $\mathrm{PhD},{ }^{\mathrm{e}}$ Christodoulos S. Flordellis, $\mathrm{PhD},{ }^{\mathrm{f}}$ and \\ Dimitrios Dougenis, $\mathrm{PhD}^{\mathrm{a}}$
}

\begin{abstract}
Objective: Thrombin has been reported to play a pivotal role in the initiation of angiogenesis by indirectly regulating and organizing a network of angiogenic molecules. On the basis of these reports, we investigated the angiogenic action of thrombin in a rabbit model of acute myocardial infarction.
\end{abstract}

Methods: A rabbit model of acute myocardial infarction was established by ligation of the left anterior descending
coronary branch. Subjects were then divided into 2 groups and treated with intramyocardial administration of
thrombin ( $2500 \mathrm{IU} ; \mathrm{n}=13$ ) or an equal volume of normal saline $(\mathrm{n}=13)$. Four weeks later, animals were eutha-
nized and histopathologic analysis, immunohistochemical staining for endothelial markers CD31 and vascular
endothelial growth factor-A, and electron microscopy examination were performed on excised hearts. Electrocar-
diography, cardiac enzymes, and assessment of cardiac function by measuring left ventricular end-diastolic
pressure and ejection fraction were recorded before and after myocardial infarction, and both left ventricular
end-diastolic pressure and ejection fraction were further measured on the day of euthanasia ( $\mathrm{n}=5-8$ in each case).

Results: Increased levels of troponin, ST elevation, and histopathologically confirmed myocardial infarction were observed in all animals. A significant increase of microvessel density at the infarct border zone, as evaluated by CD31 immunohistochemistry, was observed in the thrombin-treated group compared with the control group $(30.3 \pm 12.8$ vs $12.6 \pm 4.8, P=.0065)$. A significantly higher number of vascular endothelial growth factor-A-positive vessels at the infarct border zone was observed in the thrombin-treated animals compared with the control group $(21.8 \pm 8.9$ vs $5.6 \pm 4.4 ; P=.0009)$. The thrombin-treated animals showed a statistically significant reduction in left ventricular end-diastolic pressure values $(6.9 \pm 1.8 \mathrm{~mm} \mathrm{Hg}$ vs $12.7 \pm 2.2 \mathrm{~mm} \mathrm{Hg}$, $P=.0002)$ and significant improvement in left ventricular ejection fraction $(59.8 \% \pm 3.1 \%$ vs $42.2 \% \pm 6.14 \%$, $P=.002)$ on the day of euthanasia compared with the post-infarct day, reflecting significantly improved cardiac function compared with control subjects that showed no significant change.

Conclusions: Intramyocardial administration of thrombin seems to promote angiogenesis and improve cardiac function of the ischemic myocardium, which may provide a new therapeutic approach in patients with myocardial ischemia. (J Thorac Cardiovasc Surg 2014;147:1376-83)

Coronary artery disease is the major cause of death in the western world and an emerging health problem in developing countries. ${ }^{1,2}$ Myocardial ischemia is one of the most

From the Department of Cardiothoracic Surgery, ${ }^{\mathrm{a}}$ Patras University Hospital, School of Medicine, Rion, Greece; Department of Interventional Radiology, ${ }^{\mathrm{b}}$ Patras University Hospital, School of Medicine, Rion, Greece; Department of Anatomy-Histology-Embryology, ${ }^{c}$ Medical School, University of Patras, Rion, Greece; Department of Microbiology, ${ }^{\mathrm{d}}$ Patras University Hospital, School of Medicine, Rion, Greece; Laboratory of Histology and Embryology, ${ }^{\mathrm{e}}$ Medical School, University of Athens, Goudi, Greece; and Department of Pharmacology, ${ }^{\mathrm{f}}$ Medical School, University of Patras, Rion, Greece.

This work was supported in part by University of Patras, Research Programme "K. Karatheodori." Sofoklis Mitsos was supported by a scholarship for doctoral studies of Alexander S. Onassis Public Benefit Foundation, Athens, Greece.

Disclosures: Authors have nothing to disclose with regard to commercial support.

Received for publication June 17, 2012; revisions received May 9, 2013; accepted for publication May 16, 2013; available ahead of print July 15, 2013.

Address for reprints: Sofoklis Mitsos, PhD, Valtetsiou 3, Vrilissia, 15235, Athens, Greece (E-mail: sophocmit@yahoo.gr).

$0022-5223 / \$ 36.00$

Copyright (c) 2014 by The American Association for Thoracic Surgery

http://dx.doi.org/10.1016/j.jtcvs.2013.05.036 extensively studied topics in modern cardiovascular research. The open-chest animal platform is one of the most well-developed models of myocardial infarction in modern laboratories of cardiovascular research. ${ }^{3}$ Despite major advances in the prevention and treatment of atherosclerotic vascular disease, a large number of patients with advanced symptomatic coronary artery disease have disabling symptoms despite pharmacotherapy and revascularization by coronary artery bypass grafting or percutaneous transluminal coronary angioplasty. It is well known that coronary collateral vessels protect myocardial tissue from ischemic injury. ${ }^{4}$ Therapeutic myocardial angiogenesis is a novel approach based on the development of new blood vessels in ischemic regions under the influence of the appropriate growth factors. ${ }^{5}$ Treatment of coronary disease using growth factors that augment vessel growth is increasingly proposed for otherwise untreatable patients either alone or in combination with other procedures. Successful preclinical studies and promising early results in clinical trials have 


\author{
Abbreviations and Acronyms \\ AMI = acute myocardial infarction \\ CAM = chick chorioallantoic membrane \\ ECG = electrocardiography \\ IM = intramuscularly \\ LAD $=$ left anterior descending \\ LVEDP $=$ left ventricular end-diastolic pressure \\ LVEF = left ventricular ejection fraction \\ MVD = microvessel density \\ $\mathrm{PB}=$ phosphate buffer \\ VEGF = vascular endothelial growth factor
}

created excitement about the potential of coronary therapeutic angiogenesis. ${ }^{6}$ Many angiogenic growth factors have been identified, but few have been tested in clinical trials and most trials have focused on vascular endothelial growth factor (VEGF) and fibroblast growth factor with controversial results. ${ }^{5-7}$

Apart from its central role in blood coagulation, thrombin is also claimed to be a potent activator of angiogenesis. The angiogenic action of thrombin has been demonstrated in the chick chorioallantoic membrane (CAM) system ${ }^{8}$ and the in vivo Matrigel system. ${ }^{9}$ This is a thrombin receptor-mediated event and is independent of fibrin formation. Thrombin, unlike other angiogenic factors, has a multiplicity of effects on angiogenesis and plays a significant role in regulating vascular functions, vascular stability, promotion of endothelial cell survival and interaction with other factors, and many cell types. Consequently, thrombin may have the unique ability to orchestrate the requirements for the formation of mature blood vessels and may have therapeutic angiogenic applications. In the present study, we investigated the effects of direct intramyocardial administration of thrombin and its ability to induce vessel growth in a rabbit model of acute myocardial infarction (AMI).

\section{MATERIALS AND METHODS}

This investigation was carried out in conformity with the "Guide for the Care and Use of Laboratory Animals" published by the National Institutes of Health (publication No. 86-23, revised 1985) and approved by the local University Hospital's Scientific and Ethical Committee. New Zealand White rabbits weighing 2.5 to $3.5 \mathrm{~kg}$ were kept in separate cages in an environmentally controlled animal research facility. The experimental protocol included 2 groups: (1) a group of animals that underwent tracheostomy, subxiphoid incision, left anterior descending (LAD) artery ligation with suture, and intramyocardial administration of thrombin, and (2) a control group of animals that underwent tracheostomy, subxiphoid incision, LAD artery ligation with suture, and intramyocardial administration of saline $0.9 \% \mathrm{NaCl}$.

\section{Experimental Model of Acute Myocardial Infarction}

All procedures were performed with the animals under anesthesia, with a mixture of ketamine $(35 \mathrm{mg} / \mathrm{kg})$ and xylazine $(5 \mathrm{mg} / \mathrm{kg})$ administered intramuscularly (IM). ${ }^{10}$ Subsequent anesthesia was maintained with low doses of propofol. The surgical procedure was performed in a sterilized fashion. The subxiphoid region was clipped and shaved, followed by sterile surgical draping. A 22-gauge intravenous catheter was inserted into the marginal ear vein for venous access. The animals were placed on a heating pad in the supine position, and an intravenous normal saline drip $(15 \mathrm{~mL} / \mathrm{h})$ was established.

Immediately before ligation, $1 \mathrm{mg} / \mathrm{kg}$ of lidocaine was administered intravenously to minimize potential ventricular arrhythmias. A prophylactic antibiotic (cefuroxime $35 \mathrm{mg} \mathrm{IM}$ ) was administered before and after surgery, and an analgesic was given postoperatively. Electrodes for electrocardiography (ECG) were attached in both the front limbs and the lower abdomen at bilateral positions. Arterial oxygen saturation was measured with a standard pulse oximeter. The ECG, systemic blood pressure, and pulse oximetry were monitored continuously during surgery. A tracheostomy was performed, and subjects were ventilated with room air through a $3.5-\mathrm{mm}$ pediatric tracheal tube at a rate of 35 to 40 breaths $/ \mathrm{min}$ and a tidal volume of 10 to $15 \mathrm{~mL}$ using room air enriched with oxygen. The tracheostomy tube was removed after the end of the experimental surgical procedure.

To mimic clinically relevant coronary artery disease, we used a model of myocardial infarction. A skin incision was made over the subxiphoid region and sternum. The xiphoid process was carefully detached from the sternal part of diaphragm. A mini median sternotomy was performed carefully along the midline to avoid injury to the parietal pleura. ${ }^{11,12}$ The sternal edges were spread, and an incision was made at the cardiac sac to expose the myocardial ventricular wall. A 5-0 monofilament polypropylene suture was placed around the LAD coronary artery approximately 8 to $10 \mathrm{~mm}$ from its origin. Within 5 minutes after coronary occlusion, rabbits were divided into 2 groups. In the thrombin group, bovine-derived thrombin (FloSeal; Baxter Healthcare Corp, Vienna, Austria) was injected using a thin 27-gauge needle into the myocardium at the ischemic area of the left ventricular wall. Thrombin $2500 \mathrm{IU}$ was diluted in $0.5 \mathrm{~mL}$ of solution and administered slowly with 5 separate injections $(0.1 \mathrm{~mL}$ per injection). Injections were equally distributed along the ischemic myocardium with a radius of a few millimeters between each other.

There were no changes in blood pressure or heart rate associated with the injection of thrombin and no obvious adverse effects, such as anaphylactic reaction, in rabbits throughout the experiment. In control rabbits, an equal volume of saline $(0.5 \mathrm{~mL})$ was injected in the same manner. After hemodynamic stability, the sternum, muscle layers, and skin were closed, and rabbits were allowed to recover. During surgery, an 18-gauge catheter was inserted into the left ventricle via the left ventricular apex for continuous measurement of left ventricular end-diastolic pressure (LVEDP) and was removed after successful induction of myocardial infarction. Twodimensional and M-mode echocardiographic images were obtained via a right parasternal approach to determine the ejection fraction because the best acoustic window could be acquired from the right parasternal position that provided long and short-axis views of the left ventricle equivalent to the left parasternal window in humans. Serum levels of aspirate aminotransferase, lactate dehydrogenase, creatine kinase, and MB isoenzyme of creatine kinase and troponin were measured with a microparticle enzyme immunoassay (MEIA AxSYM; Abbott Laboratories, Abbott Park, IIl) and Liaison assay before and 3, 6, 12, and 24 hours after ligation to assess irreversible myocardial injury invoked during the procedure.

\section{Histopathologic Analysis}

Four weeks after ligation of the LAD artery, the rabbits were anesthetized again with ketamine $(35 \mathrm{mg} / \mathrm{kg})$ and xylazine $(5 \mathrm{mg} / \mathrm{kg}) \mathrm{IM}$. A median sternotomy was performed, and adhering tissue was carefully removed around the myocardium. Heparin $(1000 \mathrm{U})$ was given intravenously to prevent clotting. An 18-gauge catheter was reintroduced through the ventricular apex, and LVEDP measurements were taken before euthanasia as described previously. All subjects were then euthanized with an overdose of propofol, and hearts were excised along with the aortic root. 
Excised hearts were examined macroscopically for gross changes because the infarct area is easily identified and delineated macroscopically 4 weeks after the ischemic injury. ${ }^{13,14}$ After macroscopic examination, 3-mm-thick sections were taken at the greatest dimension of clearly identifiable "graywhite" post-infarct fibrotic areas, fixed in $10 \%$ formalin, and embedded in paraffin. Sections $(4 \mu \mathrm{m})$ from paraffin-embedded blocks were stained with hematoxylin-eosin and Masson trichrome staining for histopathologic analysis.

\section{Immunohistochemistry}

Anti-CD31 is a widely used and reliable method for assessing angiogenesis. ${ }^{15,16}$ Immunohistochemical analysis was performed using a standard biotin-streptavidin-peroxidase method. Sections $(4 \mu \mathrm{m})$ from paraffinembedded blocks were subjected to de-paraffinization, rehydration, and antigen epitope retrieval with DAKO target retrieval solution $\mathrm{pH} 9$ in a pressure cooker (pretreatment; DAKO, Glostrup, Denmark) for 15 minutes at $90^{\circ} \mathrm{C}$. Endogenous peroxidase activity was blocked with $1 \%$ hydrogen peroxide for 15 minutes. To reduce nonspecific background staining, slides were incubated with protein-blocking solution (goat serum) for 10 minutes. Sections were then incubated with the mouse monoclonal anti-CD31 antibody (endothelial cell clone JC70A, DAKO) at 1:40 dilution for 30 minutes at room temperature and mouse monoclonal anti-VEGF (BD Biosciences, Franklin Lakes, $\mathrm{NJ}$ ) dilution 1:50 overnight at $4{ }^{\circ} \mathrm{C}$. Bound primary antibody was detected using the UltraVision horseradish peroxidase mouse (Neomarkers; Lab Vision, Fremont, Calif) according to the manufacturer's instructions. Diaminobenzidine was used as the chromogen for visualization. Slides were counterstained with hematoxylin, dehydrated, and mounted. To test the specificity of the antibody, the same protocol was applied by replacing the primary antibody with protein blocking solution (negative control). ${ }^{15-17}$ For the assessment of microvessel density (MVD) based on CD31 immunostaining, infarct area, border zone, and remote area in each case were evaluated separately. The areas of highest vascularization (hot spots) were chosen at low power $(\times 100)$ and microvessel counting followed on 3 chosen high-power fields. The recorded MVD was the median of the vessel counts obtained in these 3 fields. Counting of vessels positive for VEGF-A expression was performed similar to MVD.

\section{Transmission Electron Microscopy}

Glutaraldehyde, propylene oxide, epoxy resins, and lead citrate were obtained from Serva (Heidelberg, Germany). The salts for the preparation of phosphate buffer (PB) and the absolute ethanol were obtained from Merck (Whitehouse Station, NJ). Uranyl acetate and osmium tetroxide were obtained from Polyscience (Warrington, Pa). The diamond knife was obtained from Diatome Ltd (Biel, Switzerland). All the photographic material, such as films, developer, and fixer solutions, were obtained from Kodak (New York, NY).

Tissue specimens were collected immediately after the subjects were killed. Small pieces of the tissues $\left(\sim 1.5-2 \mathrm{~mm}^{3}\right)$ were cut and immediately immersion-fixed for 3 hours at $4{ }^{\circ} \mathrm{C}$ in Karnovsky fixative containing 3\% glutaraldehyde, $1 \%$ formaldehyde in PB $0.1 \mathrm{~mol} / \mathrm{L}, \mathrm{pH} 7.4$. The tissue pieces were washed in 3 changes of $\mathrm{PB}$ and post-fixed in $2 \%$ aqueous osmium tetroxide for 2 hours at $4^{\circ} \mathrm{C}$. The specimens were then dehydrated with increasing concentrations of ethanol (for 10 minutes each in $25 \%$, $50 \%, 70 \%$, and $95 \%$ ethanol and for 10 minutes in $100 \%$ ethanol twice), followed by propylene oxide ( 2 changes, 5 minutes each), infiltrated gradually in a mixture of Epon/Araldite resins diluted in propylene oxide $(1: 2,1: 1,2: 1,1$ hour each) and then embedded in fresh epoxy resin mixture. Finally, the specimens were allowed to polymerize at $60^{\circ} \mathrm{C}$ for 24 hours in an oven.

For electron microscopy, ultrathin (80-90 nm thickness) sections were cut with a Diatome Ltd diamond knife on a Leica (Wetzlar, Germany) Ultracut R ultramicrotome and collected onto 200-mesh uncoated copper grids. The grids were counterstained with saturated ethanolic uranyl acetate and lead citrate. Sections were viewed in a Zeiss EM 900 (Carl Zeiss Meditec, Jena, Germany) electron microscope, at $80 \mathrm{kV}$ accelerating voltage, with an objective aperture of $30 \mu \mathrm{m}$. The electron micrographs were recorded on Kodak Electron Image film SO-163 (gelatin plates, $8 \mathrm{~cm} \times 10$ $\mathrm{cm}$, Code No: 1679257) and then developed with the Kodak D-19 developer. The negatives were scanned with a Mikrotek (Santa Fe Springs, Calif) ScanMaker 9800XL scanner using optical resolution of $1000 \mathrm{ppi}$ and stored as tiff files. When required, the digitized images were printed at $1200 \mathrm{dpi}$ on an HP Laserjet 4250n high-resolution laser printer (Hewlett Packard Co, Palo Alto, Calif).

\section{Statistical Analysis}

Discrete variables were provided as counts and percentages, and continuous variables were given as medians and interquartile ranges (25th-75th percentiles) or as means \pm standard deviation if they originated from normal distributions. The unpaired Student $t$ test was used to test the significance of difference of variables that passed the normality test. The chi-square test was used for testing of qualitative variables, and the Mann-Whitney test was used for nonparametric testing of continuous variables that did not pass the Kolmogorov-Smirnov normality test.

\section{RESULTS \\ Electrocardiography and Blood Biochemistry}

ECG of all rabbits was normal on baseline. ST-segment elevation, an indicator of severe ischemia or ongoing infarction of full-thickness myocardium, was seen in each animal almost immediately after occlusion of the LAD artery. In total, 21 of 24 animals survived after suture placement around the LAD coronary artery producing total LAD occlusion. Another 5 animals died after being randomized to a treatment group. There were 3 deaths in the thrombin group (3/11) and 2 deaths in the control group (2/10). Two of the subjects died of ventricular fibrillation after quick intramyocardial injections (1 control animal and 1 thrombin animal). The next 2 deaths were recorded 24 hours after intramyocardial administration (1 control subject and 1 thrombin subject). One rabbit in the thrombin group had a late death within the first 10 days after myocardial infarction due to sepsis. Overall experimental mortality rate was $33.3 \%(8 / 24)$, and as a result 8 subjects were included in each study arm. Four weeks after myocardial infarction, ECG showed abnormal Q waves in all surviving animals. There was no statistically significant difference in the ST-elevation between the 2 groups (Figure 1).

Myocardial ischemia was confirmed with a significant increase of aspirate aminotransferase, lactate dehydrogenase, creatine kinase, MB isoenzyme of creatine kinase, and tropo$\operatorname{nin} \mathrm{T}$ after occlusion of coronary artery compared with baseline values. There was no statistically significant difference in myocardial-enzyme changes in blood serum 24 hours after LAD occlusion between the 2 groups (data not shown). Serum troponin-T levels before induction of infarction were less than $0.2 \mathrm{ng} / \mathrm{mL}$ in both groups. Troponin-T levels were significantly increased 24 hours after coronary occlusion in both the thrombin and control groups, but there was no significant difference between the 2 groups $(49.6 \pm 65.1 \mathrm{ng} / \mathrm{mL}$ vs $40.0 \pm 57.1 \mathrm{ng} / \mathrm{mL}$, respectively, $P=.76$; Figure 1 ). 


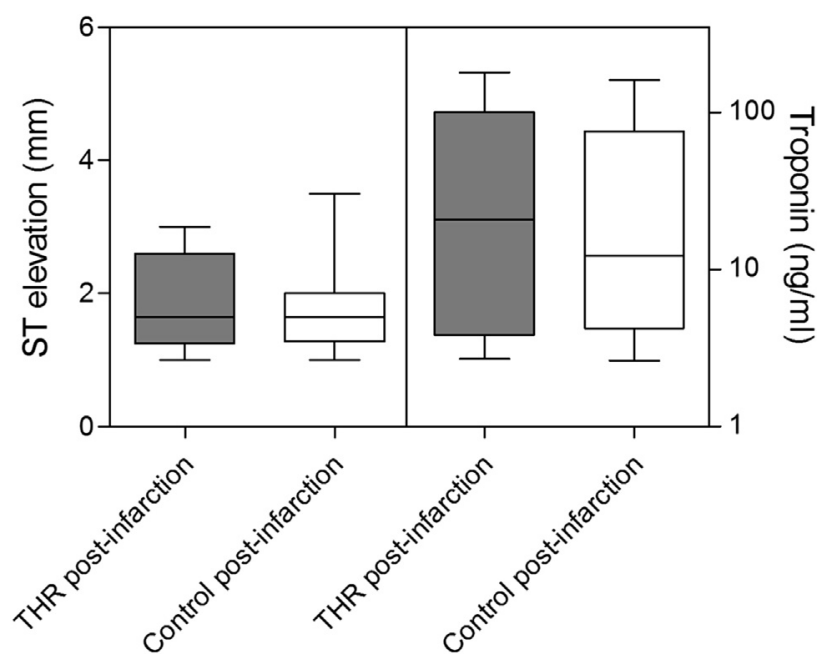

FIGURE 1. ECG and troponin. Myocardial ischemia was confirmed by ST-segment elevation on ECG in all subjects. There was no statistically significant difference between the 2 groups with regard to ST-segment elevation (left) or troponin T levels at 24 hours (right). THR, Thrombin-treated group.

\section{Histologic Examination}

Histolopathologic analysis of heart sections confirmed the presence of myocardial infarction in both groups. Granulation tissues with new blood vessels, infiltration by lymphocytes, plasma cells, histiocytes, and collagen deposition were identified at the site of myocardial infarction. Infarct size was measured on sections stained with hematoxylin-eosin and Masson trichrome that were taken at the greatest dimension of macroscopic gray-white areas (Figure 2). Myocardial infarct size was $0.8 \pm 0.4 \mathrm{~cm}$ (range, $0.6-1.7 \mathrm{~cm})$ in the thrombin-treated group and $0.9 \pm 0.2 \mathrm{~cm}$ (range, 0.6-1.2 cm) in the control group. There was no statistically significant difference in the size of the myocardial infarct area between the 2 groups $(P=.49)$. Given the hypothesis that the tissue stimulus for angiogenesis is more intense in tissues adjacent to the infarct zone, MVD was examined in the border zone of the infarct with antiCD31 immunostaining. Recorded MVD was significantly higher in the infarct border zone of thrombin-treated hearts and numerically more than double the one recorded in the control group $(30.3 \pm 12.8$ vs $12.6 \pm 4.8$, respectively; $P=.0065$ ) (Figure 3). VEGF-A expression was observed in endothelial cells and macrophages. The recorded number of VEGF-A positive vessels at the infarct border zone was significantly higher in thrombin-treated animals compared with the control group $(21.8 \pm 8.9$ vs $5.6 \pm 4.4$; $P=.0009$; Figure 2).

\section{Transmission Electron Microscopy}

In samples distant to infarction, myocytes appeared nearly normal. Sarcomeres showed a normal pattern, and most of the mitochondria were oval with a granular, electron-dense matrix with typical orderly arranged cristae. Signs of ischemic injury were not visible (Figure $4, A$ ). On the contrary, in ischemic areas, extended intracellular
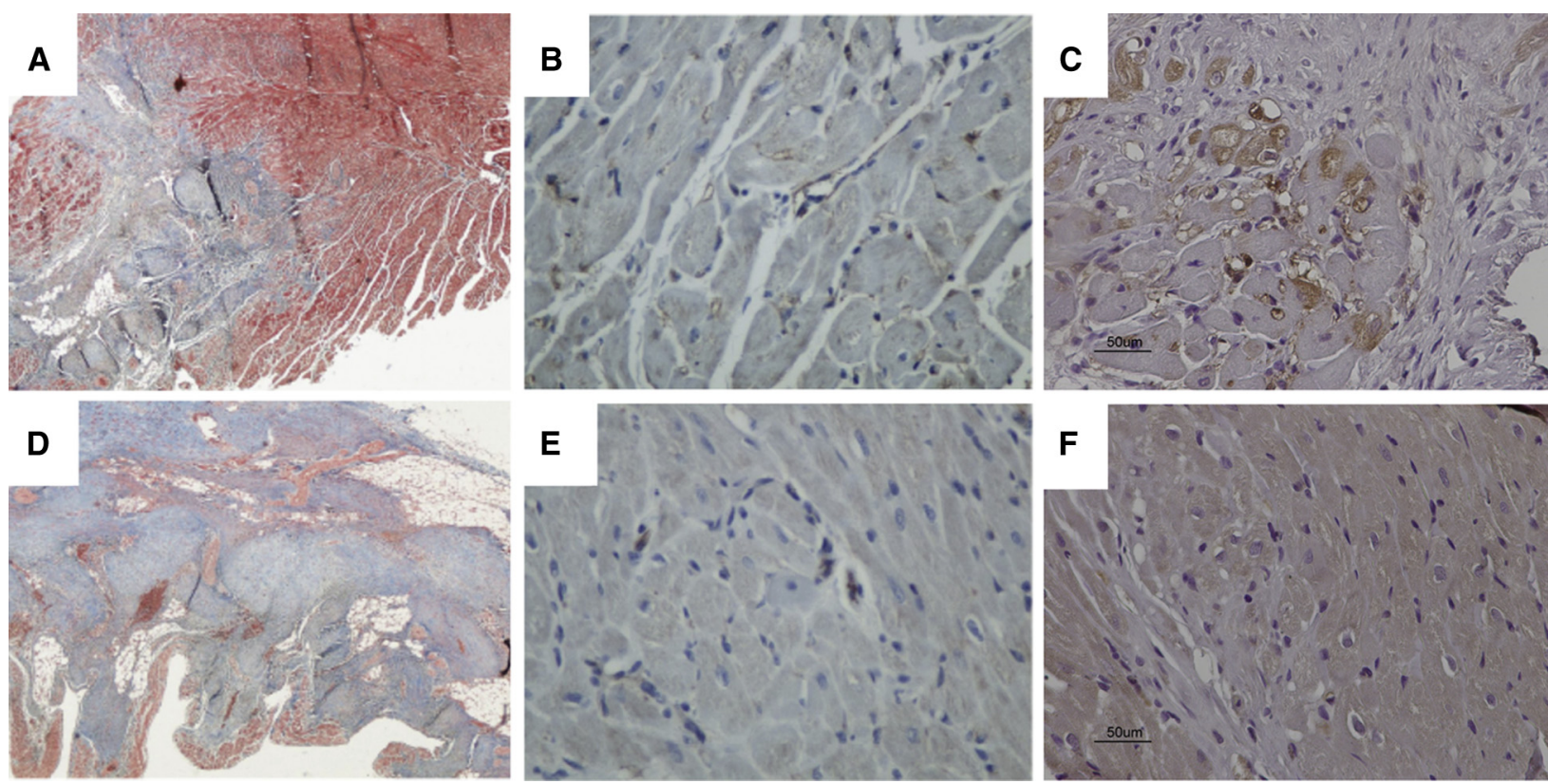

FIGURE 2. Histologic examination. Masson trichrome staining (A and D) of myocardial infarct in the 2 groups. Representative cases of thrombin-treated (A; top) and control group (D; bottom) are presented (magnification $\times 40$ ). CD31-immunostaining of vessels at the infarct border zone for MVD measurements. Representative cases of thrombin-treated (B; top) and control group (E; bottom) (magnification $\times 400)$. VEGF-A expression in vessels at the infarct border zone. Representative cases of thrombin-treated (C; top) and control group subjects (F; bottom) (magnification $\times 400)$. 


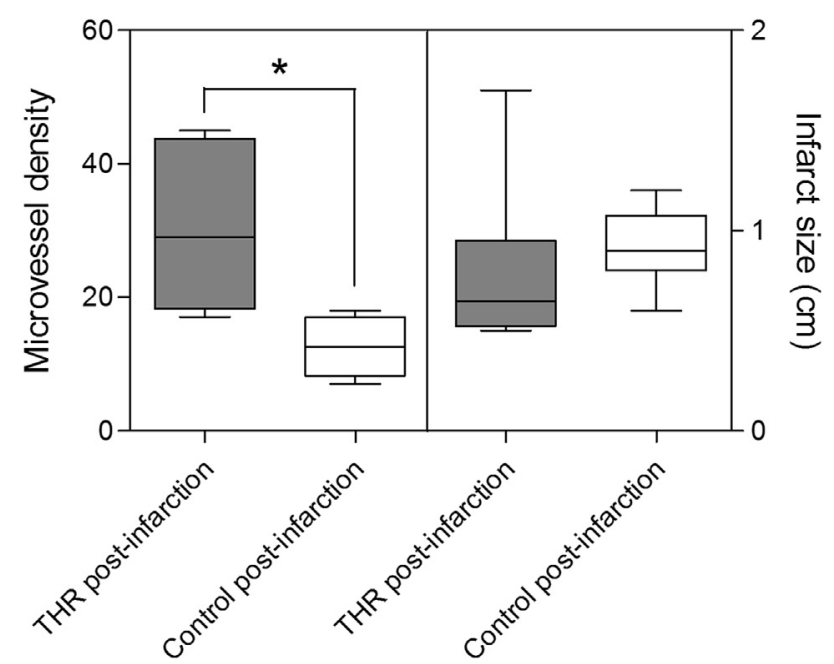

FIGURE 3. MVD and infarct size. MVD measured at the infarct border zone was significantly higher in the hearts treated with 2500 IU thrombin compared with the saline-treated control group (left; $* P<.05$ ). Histomorphometry of the myocardial infarct area shows a similar extent of necrosis in the 2 groups (right). THR, Thrombin-treated group.

edema was observed. Sarcomeres were perturbated and almost completely disorganized, and mitochondria appeared as dense irregular structures with disordered fragments of cristae (Figure 4, B). After myocardial infarction, a mild injury was visible in the border zone of the thrombin-treated group. Tissue consisted of a more or less regular pattern of sarcomeres with I-bands misaligned to a significant extent and slightly disordered mitochondria with dense grainy matrix. A slight intracellular edema was seen. High-resolution electron micrographs showed that in the border zone of thrombin-treated hearts, signs of neovascularization were visible by the formation of small extensions of the capillary walls (Figure 4,C). Untreated tissues appeared to have significant damage in the border zone with disordered myofilaments exhibiting signs of contraction or relaxation, but compared with the thrombin-treated samples, they showed less signs of neovascularization (Figure 4,D).

\section{Hemodynamic Measurements}

Cardiac function was assayed by recording LVEDP and left ventricular ejection fraction (LVEF), which were measured before and after myocardial infarction and on the day of euthanasia, offering an indication of left ventricular systolic function. The main effects of infarction were a significant increase of LVEDP and significant decrease of LVEF in all infarcted subjects because of myocardial dysfunction. At baseline, LVEDP and LVEF were $7.1 \pm 1.7 \mathrm{~mm} \mathrm{Hg}$ and $68.2 \% \pm 4.7 \%$, respectively, in the thrombin group, and $6.7 \pm 1.3 \mathrm{~mm} \mathrm{Hg}$ and $69.6 \% \pm 3.8 \%$, respectively, in the control group. LVEDP and LVEF changed to $12.7 \pm 2.2$ $\mathrm{mm} \mathrm{Hg}$ and $42.2 \% \pm 6.1 \%$, respectively, in the thrombin group, and to $12.2 \pm 2.3 \mathrm{~mm} \mathrm{Hg}$ and $42.8 \% \pm 7.2 \%$, respectively, in the control group soon after induction of AMI. However, on the day of euthanasia the thrombintreated animals showed a statistically significant reduction in LVEDP $(6.9 \pm 1.8 \mathrm{~mm} \mathrm{Hg}$ vs $12.7 \pm 2.2 \mathrm{~mm} \mathrm{Hg}$, $P=.0002)$ and significant increase in LVEF (59.8 \pm $3.1 \%$ vs $42.2 \pm 6.1 \%, P=.002)$ compared with the post-infarct day, reflecting recovery of cardiac function most likely due to increased regional coronary angiogenesis, whereas no change was noted in the control group (Figures 5 and 6).

\section{DISCUSSION}

In the present study, a single intramyocardial injection of thrombin in a rabbit model of AMI enhanced the angiogenic response to ischemia with a significant increase of regional collateralization. These benefits were accompanied by improvements in cardiac systolic function in the ischemic territory. New collaterals were developed to protect the ischemic myocardium from injury. This native arteriogenic response was profoundly augmented by thrombin with a statistically significant increase of MVD and VEGF expression compared with the control group. Although there was not a significant decrease of infarct size in the thrombin group, a statistically significant restoration of heart function as indicated by reduction of LVEDP and increase of ejection fraction in the thrombin group was noted. The improvement in cardiac function probably resulted from a combination of factors, including improvement of local myocardial perfusion and contractility. In theory, the contractile function of hibernating myocardium may be improved by the improvement of myocardial perfusion with angiogenesis. To our knowledge, this is the first experimental study to investigate the angiogenic action of thrombin in the acutely ischemic myocardium.

Our results are in line with previously described effects of thrombin administration and build further on previous reports indicating that thrombin functions as a potent proangiogenic molecule. Tsopanoglou and colleagues ${ }^{8}$ suggested that thrombin can promote angiogenesis in the CAM system and that the functional catalytic site of thrombin is required. The ability of thrombin to stimulate the formation of vessels has been shown in the Matrigel in vivo model. ${ }^{9}$ Moreover, Dimitropoulou and colleagues ${ }^{18}$ reported that thrombin caused significant increases in the numbers, diameters, and lengths of capillaries in the CAM system. Many cellular effects of thrombin on endothelial cells can contribute to the angiogenic action of thrombin. ${ }^{19,20}$ Thrombin decreases the ability of endothelial cells to attach to basement membrane proteins. Thrombin upregulates the expression of $\alpha \mathrm{v} \beta 3$ integrin, ${ }^{21,22}$ which is the marker of the angiogenic phenotype of endothelial cells and serves as a ligand to this receptor. ${ }^{23}$ Furthermore, thrombin increases the secretion of VEGF and enhances the expression 

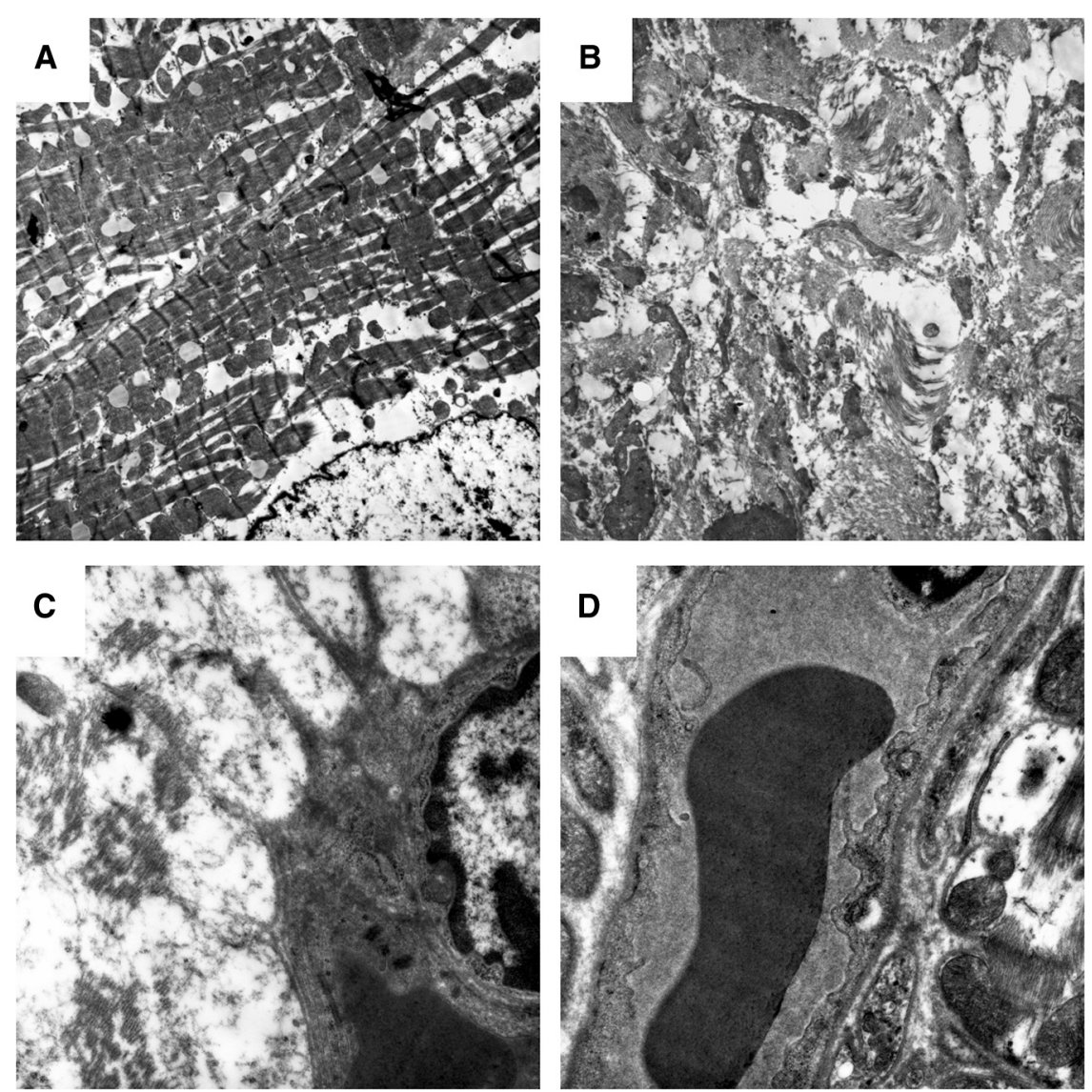

FIGURE 4. Transmission electron microscopy. Examples images of (A) remote zone and (B) infarct area after experimental myocardial infarction in the New Zealand White rabbit. Representative transmission electron microscopy images of (C) capillary sprouting on the border zone of the thrombin group, compared with images of (D) capillaries on the border zone of the control group. Note the improved neovascularization in the infarct border-zone of thrombin-treated rabbit hearts compared with saline-treated control cases (C and D).

and protein synthesis of matrix metalloproteases. ${ }^{19,24}$ Moreover, thrombin has chemotactic and haptotactic effects on endothelial cells and upregulates the expression of VEGF receptors (kinase insert domain-containing receptor and fms-like tyrosine kinase). ${ }^{23-25}$ Thus, thrombin synergizes with the key angiogenic factor VEGF in endothelial cell proliferation. Thrombin itself and its receptor protease-activated receptor 1 orchestrate many cellular events through interaction with a variety of other factors and cell types 1 and play a potential role in the complexity of the regulatory processes involved in the angiogenic cascade. ${ }^{26,27}$ Thrombin protects endothelial cells from apoptosis through a mechanism mostly independent of its catalytic active site and PAR1 activation, whereas the integrins $\alpha \nu \beta 3$ and $\alpha 5 \beta 1$ play an essential role in thrombin-induced cytoprotection. ${ }^{28}$ Katsanos and colleagues ${ }^{29}$ have recently shown that thrombin promoted arteriogenesis and improved the perfusion of distal ischemic tissue in a rabbit hind-limb ischemia model. It was demonstrated that a single intramuscular injection of thrombin caused a significant increase of regional collateralization and enhanced distal blood flow circulation, and resulted in both angiographically and functionally better vasculature in the rabbit hind-limb ischemic model. ${ }^{29}$

The choice of thrombin concentration dose (2500 IU) was based on its mean in vitro effective doses multiplied by 1000 . Our aim was to evaluate the effect of thrombin in reasonable dose range from a clinical viewpoint. Thrombin was administered intramyocardially with 5 separate injections. An advantage of intramyocardial administration of thrombin may be that it strictly targets the area in need, induces local angiogenesis, and seems to avoid high levels of circulating angiogenic activity that could stimulate plaque angiogenesis or growth of occult neoplasms. Opinions differ as to whether angiogenesis can be caused by a mere needle puncture of the myocardium. Mere mechanical needling of the ischemic myocardium can induce vessel growth and maintain function as shown by $\mathrm{Chu}$ and colleagues, ${ }^{30}$ whereas Horvath and colleagues ${ }^{31}$ did not observe this effect. Both experiments were done in porcine models of myocardial ischemia in the context of transmyocardial laser revascularization. In a clinical study, Pecher and 


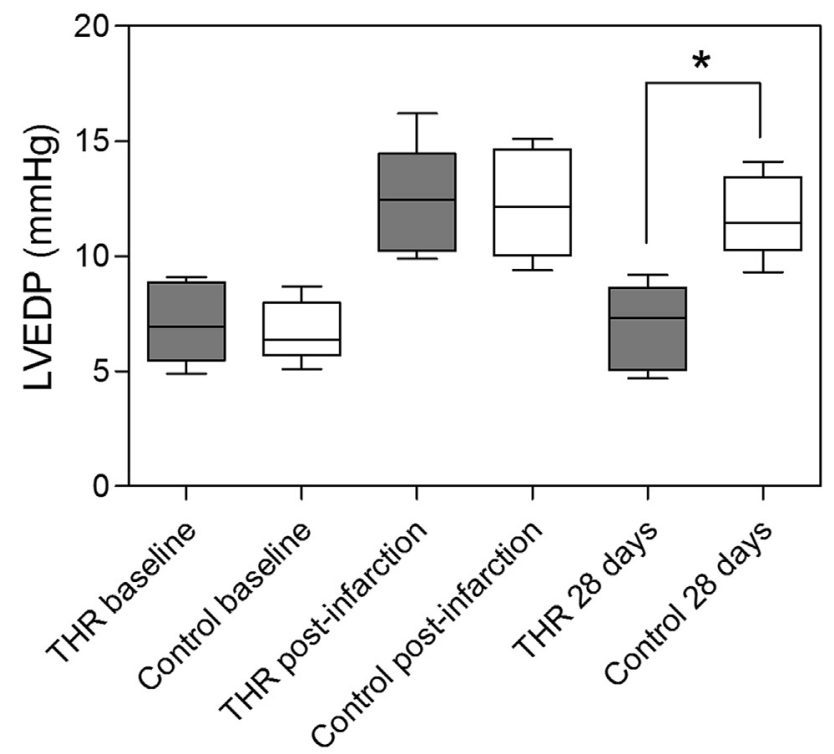

FIGURE 5. LVEDP measurements ( $\mathrm{mm} \mathrm{Hg}$ ). Induction of AMI produced a significant increase in LVEDP in both groups because of a disrupted cardiac systolic function. Four weeks post-infarction, restoration of cardiac systolic function was noted in the thrombin-treated hearts as evidenced by normalization of the recorded LVEDP compared with no significant change in the control group $(* P<.05)$. LVEDP, Left ventricular enddiastolic pressure; $T H R$, thrombin-treated group.

Schumacher ${ }^{32}$ injected intramyocardial fibroblast growth factor-1 or the heat denatured factor for control purposes. The results showed the superiority of the active protein. In the present study, both thrombin and saline were administered inside the myocardium using a thin $27 \mathrm{G}$ needle exactly in the same manner. It is certain that different agents require different applications to function optimally, and the most effective application method might even differ for different growth factors depending on their cellular target.

\section{Study Limitations}

First, thrombin was administered immediately after induction of AMI. In the clinical setting, most patients receive surgical procedures over the course of hours. It is necessary to confirm the efficacy of thrombin administered at a longer time after infarction. Moreover, it is possible that repeated multiple injections of smaller doses or sustained release of thrombin from biodegradable systems would be better than bolus injection of high doses because new collateral vessels require up to 20 days after the ischemic insult to fully develop. Furthermore, we only counted the vessels and did not measure their diameter. Therefore, additional restructuring of small arterioles to larger vessels could have eluded our observations. We did not evaluate histologic specimens for microvascular thrombosis, but there were no clinical sequelae to raise suspicion about a prothrombotic status.

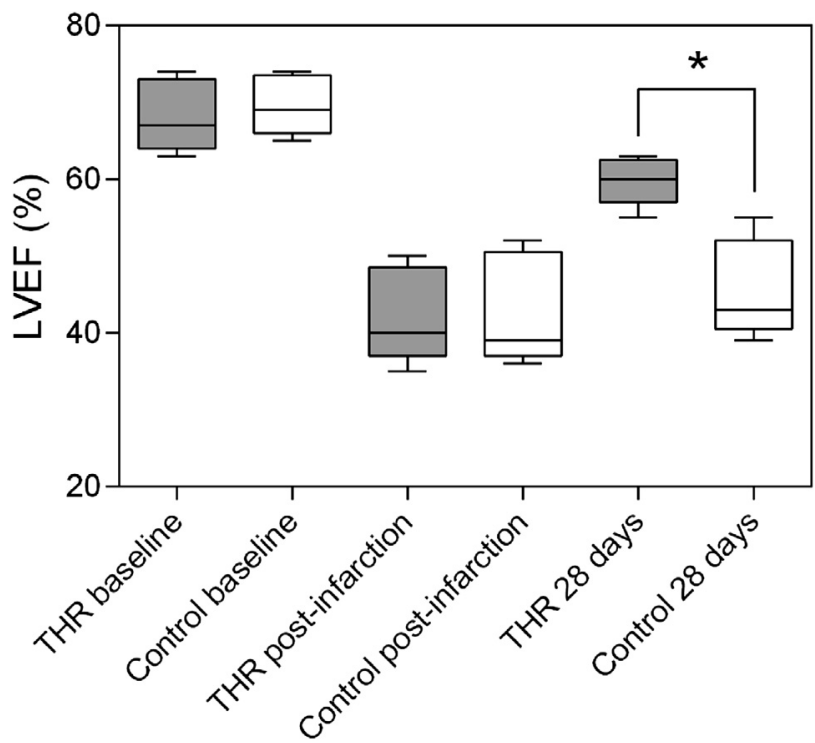

FIGURE 6. LVEF measurements (\%). Induction of AMI produced a significant decrease of LVEF in both groups because of cardiac dysfunction. Four weeks post-infarction, restoration of cardiac systolic function was noted in the thrombin-treated hearts as evidenced by partial restoration of the recorded LVEF compared with no significant change in the control group $(* P<.05)$. LVEF, Left ventricular ejection fraction; THR, thrombin-treated group.

\section{CONCLUSIONS}

This study demonstrated the ability of locally administered thrombin to induce new vessel growth and enhance cardiac function when applied intramyocardially in a rabbit model of AMI. Further studies are warranted to look into the therapeutic potential of thrombin in the setting of chronic or acute myocardial ischemia by inducing therapeutic angiogenesis and collateralization.

\section{References}

1. Murray CJ, Lopez AD. Mortality by cause for eight regions of the world: Global Burden of Disease Study. Lancet. 1997;349:1269-76.

2. Mercado N, Wijns W, Serruys PW, Sigwart U, Flather MD, Stables RH, et al. One-year outcomes of coronary artery bypass graft surgery versus percutaneous coronary intervention with multiple stenting for multisystem disease: a metaanalysis of individual patient data from randomized clinical trials. $J$ Thorac Cardiovasc Surg. 2005;130:512-9.

3. Mitsos S, Katsanos K, Dougeni E, Koletsis EN, Dougenis D. A critical appraisal of open- and closed-chest models of experimental myocardial ischemia. Lab Anim (NY). 2009;38:167-77.

4. Levin DC. Pathways and functional significance of the coronary collateral circulation. Circulation. 1974;50:831-7.

5. Mitsos S, Katsanos K, Koletsis E, Kagadis GC, Anastasiou N, Diamantopoulos A, et al. Therapeutic angiogenesis for myocardial ischemia revisited: basic biological concepts and focus on latest clinical trials. Angiogenesis. 2012;15: $1-22$.

6. Tirziu D, Simons M. Angiogenesis in the human heart: gene and cell therapy. Angiogenesis. 2005;8:241-51.

7. Chiu RC. Therapeutic cardiac angiogenesis and myogenesis: the promises and challenges on a new frontier. J Thorac Cardiovasc Surg. 2001;122: 851-2.

8. Tsopanoglou NE, Pipili-Synetos E, Maragoudakis ME. Thrombin promotes angiogenesis by a mechanism independent of fibrin formation. Am J Physiol. 1993; 264(5 Pt 1):C1302-7. 
9. Haralabopoulos GC, Grant DS, Kleinman HK, Maragoudakis ME. Thrombin promotes endothelial cell alignment in Matrigel in vitro and angiogenesis in vivo. Am J Physiol. 1997;273(1 Pt 1):C239-45.

10. Green CJ, Knight J, Precious S, Simpkin S. Ketamine alone and combined with diazepam or xylazine in laboratory animals: a 10 year experience. Lab Anim. 1981;15:163-70.

11. Fujita M, Morimoto Y, Ishihara M, Shimizu M, Takase B, Maehara T, et al. A new rabbit model of myocardial infarction without endotracheal intubation. $J$ Surg Res. 2004;116:124-8.

12. Katsanos K, Mitsos S, Koletsis E, Bravou V, Karnabatidis D, Kolonitsiou F, et al. Transauricular embolization of the rabbit coronary artery for experimental myocardial infarction: comparison of a minimally invasive closed-chest model with open-chest surgery. J Cardiothorac Surg. 2012;7:16.

13. Fishbein MC, Meerbaum S, Rit J, Lando U, Kanmatsuse K, Mercier JC, et al. Early phase acute myocardial infarct size quantification: validation of the triphenyl tetrazolium chloride tissue enzyme staining technique. Am Heart J. 1981;101:593-600.

14. Kim SJ, Kuklov A, Crystal GJ. In vivo gene delivery of XIAP protects against myocardial apoptosis and infarction following ischemia/reperfusion in conscious rabbits. Life Sci. 2011;88:572-7.

15. Kobayashi H, Yasuda S, Bao N, Iwasa M, Kawamura I, Yamada Y, et al. Postinfarct treatment with oxytocin improves cardiac function and remodeling via activating cell-survival signals and angiogenesis. J Cardiovasc Pharmacol. 2009; 54:510-9.

16. Hu X, Yu SP, Fraser JL, Lu Z, Ogle ME, Wang JA, et al. Transplantation of hypoxia-preconditioned mesenchymal stem cells improves infarcted heart function via enhanced survival of implanted cells and angiogenesis. J Thorac Cardiovasc Surg. 2008;135:799-808.

17. Angoulvant D, Fazel S, Weisel RD, Lai TY, Fedak PW, Chen L, et al. Cell-based gene therapy modifies matrix remodeling after a myocardial infarction in tissue inhibitor of matrix metalloproteinase-3-deficient mice. J Thorac Cardiovasc Surg. 2009; 137:471-80

18. Dimitropoulou C, Maragoudakis ME, Konerding MA. Effects of thrombin and of the phospholipase C inhibitor, D609, on the vascularity of the chick chorioallantoic membrane. Gen Pharmacol. 2000;35:241-7.

19. Dupuy E, Habib A, Lebret M, Yang R, Levy-Toledano S, Tobelem G. Thrombin induces angiogenesis and vascular endothelial growth factor expression in human endothelial cells: possible relevance to HIF-1alpha. J Thromb Haemost. 2003;1: 1096-102.
20. Griffin CT, Srinivasan Y, Zheng YW, Huang W, Coughlin SR. A role for thrombin receptor signaling in endothelial cells during embryonic development. Science. 2001;293:1666-70

21. Tsopanoglou NE, Andriopoulou P, Maragoudakis ME. On the mechanism of thrombin-induced angiogenesis: involvement of alphavbeta3-integrin. Am J Physiol Cell Physiol. 2002;283:C1501-10.

22. Tsopanoglou NE, Papaconstantinou ME, Flordellis CS, Maragoudakis ME. On the mode of action of thrombin-induced angiogenesis: thrombin peptide TP508, mediates effects in endothelial cells via alphavbeta3 integrin. Thromb Haemost. 2004;92:846-57.

23. Maragoudakis ME, Tsopanoglou NE, Andriopoulou P. Mechanism of thrombininduced angiogenesis. Biochem Soc Trans. 2002;30:173-7.

24. Maragoudakis ME, Tsopanoglou NE. On the mechanism(s) of thrombin induced angiogenesis. Adv Exp Med Biol. 2000;476:47-55.

25. Tsopanoglou NE, Maragoudakis ME. On the mechanism of thrombin-induced angiogenesis. Potentiation of vascular endothelial growth factor activity on endothelial cells by up-regulation of its receptors. J Biol Chem. 1999;274 23969-76

26. Tsopanoglou NE, Maragoudakis ME. Role of thrombin in angiogenesis and tumor progression. Semin Thromb Hemost. 2004;30:63-9.

27. Tsopanoglou NE, Maragoudakis ME. Thrombin's central role in angiogenesis and pathophysiological processes. Eur Cytokine Netw. 2009;20:171-9.

28. Zania P, Papaconstantinou M, Flordellis CS, Maragoudakis ME Tsopanoglou NE. Thrombin mediates mitogenesis and survival of human endothelial cells through distinct mechanisms. Am J Physiol Cell Physiol. 2008;294 C1215-26.

29. Katsanos K, Karnabatidis D, Diamantopoulos A, Kagadis GC, Ravazoula P, Nikiforidis GC, et al. Thrombin promotes arteriogenesis and hemodynamic recovery in a rabbit hindlimb ischemia model. J Vasc Surg. 2009;49: 1000-12.

30. Chu VF, Giaid A, Kuang JQ, McGinn AN, Li CM, Pelletier MP, et al. Thoracic Surgery Directors Association Award. Angiogenesis in transmyocardial revascularization: comparison of laser versus mechanical punctures. Ann Thorac Surg. 1999;68:301-8

31. Horvath KA, Belkind N, Wu I, Greene R, Doukas J, Lomasney JW, et al. Functional comparison of transmyocardial revascularization by mechanical and laser means. Ann Thorac Surg. 2001;72:1997-2002.

32. Pecher P, Schumacher BA. Angiogenesis in ischemic human myocardium: clinical results after 3 years. Ann Thorac Surg. 2000;69:1414-9. 